\section{What makes a good medical journal great?}

I was surprised to learn about the dismissal of CMAJ's editor-in-chief and the disbandment of the CMAJ's journal oversight committee. ${ }^{1}$ One of the reasons given for such actions was that the journal's reputation and revenue have been slipping. Indeed, CMAJ's impact factor has been slightly declining the past few years. However, the reputation of the journal is not just the impact factor.

The main goal of most medical journals is improving medical care by publishing sound scientific articles (both research and practice papers) and focusing on topics that are of great importance to its readership. Secondary journalistic goals include improving the impact factor and breaking hot medical news. Because publishing a medical journal is a business like any other business, there are also business goals, which include increasing revenue and receiving financial support from industry. Typically, the business goals are the prerogative of journal owners. All these incentives may be conflicting.

Given that editorial independence is valued by both authors and readers, the hiring and firing of the editors is occasionally fiercely debated because it raises questions about editorial freedom and the culture of journals. ${ }^{2}$

So, what makes a good journal great? First and foremost, there is the team of dedicated and highly qualified editors who provide valuable guidance on the journal contents. In addition, there are manuscript authors, equally important as the editors. Ultimately, they are the ones conducting research, writing and rewriting drafts, and submitting the manuscript in hopes that it will be published. Then, there are the reviewers, who advise the editors on the importance and novelty of the findings and the soundness of the research methodology. Finally, there are the readers, who look to the journal for accurate and up-to-date scientific and clinical information.

It is the editors' integrity and independence that guarantee a proper editorial process and the quality of published contents. Any interference from the journal owners in the editorial matter may greatly disrupt the fine balance of editorial independence. Surely, the dismissal of the editor-in-chief and the disbandment of the journal oversight committee leave the impression of unnecessary leverage over the journal and its editors. The current CMA actions are reminiscent of the ones that unfolded a decade ago. Such turmoil may in fact raise authors' and readers' doubts as to the editorial independence and the quality of published articles.

I hope the CMA Board will remember the words of Thomas Jefferson: "That government is best which governs least." The main thrust of the quote is the idea that government, or the owner in this instance, should not intervene in the life of its journal any more than is absolutely necessary.

\section{Bartosz Hudzik MD PhD}

Third Department of Cardiology, Silesian Center for Heart Disease, SMDZ, Medical University of Silesia, Zabrze, Poland

\section{References}

1. Kelsall D, Patrick K, Stanbrook MB, et al. Upholding the integrity of your CMAJ. CMAJ 2016;188: E113-4.

2. Rennie D. Editors and owners - stretching reputation too far. JAMA 1999;282:783-4.

CMAJ 2016. DOI:10.1503/cmaj.1150100

\section{Benefits of pharmacotherapy for preventing hip fracture}

Although falls and fractures are common in nursing homes, the guideline on preventing fracture in long-term care in $C M A J$ emphasizes therapies of unproven benefit and small effect size. ${ }^{1}$ The authors recognize that the evidence for their recommendations for pharmacotherapy in preventing fractures in long term care is weak; however, even with stronger evidence, the effect size remains very small and possibly not significant, at $2.5 \%$ for hip fracture prevention over two years.

The studies used to develop this guideline typically involved communitydwelling patients nearly 20 years younger than the average patient in a nursing home. In our homes in Calgary (Intercare Corporate Group Inc.), the average annual incidence of hip fracture over the past 9 years has been 7.2/100 000 resident days, or approximately 18 hip fractures a year across 700 beds.
For a medium-sized home (200 residents), we could therefore expect 5 hip fractures a year, or 10 hip fractures over 2 years. If, as the authors suggest, we could reduce the fractures by 25 for every 1000 residents treated (number needed to treat $=40$ ) over 2 years, we would need to treat every patient (assuming 100\% residents are at high risk and that all survive 2 years after admission) for 2 years to prevent 5 hip fractures in a 200-bed home. However, the median length of stay is 1.9 years in Calgary homes, so $50 \%$ of our patients would not survive to see a benefit at 2 years. This, in crude terms, would therefore reduce the number of "prevented hip fractures" by only 2.5.

Such a recommendation is not an attractive or realistic proposition to most patients or providers, especially without any reference to numbers needed to harm. To further complicate matters, we are poor at estimating prognosis in the nursing home setting - only really understanding that a third of our patients will die in any given year. Selecting patients who are likely to survive one year is challenging in those with endstage chronic disease such as dementia. The authors arbitrarily place a one-year decision point in Figure 1 without any rationale to support it. Also, the broad application of this guideline, as illustrated in Figure 1, makes no reference to the wishes of patients and therefore the appropriateness of the interventions.

More attention perhaps should have been given to targeted high-risk populations in nursing homes who might be expected to experience realistic benefits. We need a better understanding of which patients to treat and what the actual effect size is in the nursing home.

I look forward to the results of future research using complex, frail nursing home patients with multiple comorbidities. It goes without saying that we would dearly love to reduce our rates of hip fracture morbidity and mortality.

\section{Patrick Quail MB}

University of Calgary, Calgary, Alta.

\section{Reference}

1. Papaioannou A, Santesso N, Morin SN, et al. Recommendations for preventing fracture in long-term care. CMAJ 2015;187:1135-44.

CMAJ 2016. DOI:10.1503/cmaj.1150090 